\title{
Frequent Patterns Analysis using Apriory: A Survey
}

\author{
Madhavi G. Patil \\ Student \\ Dr. D.Y. Patil College of Engineering Pune
}

\author{
Ravi P. Patki \\ Guide \\ Dr. D.Y. Patil College of Engineering Pune
}

\begin{abstract}
In applications such as location-based services, natural habitat monitoring, web data integration, and biometric applications, the values of the underlying data are inherently noisy or imprecise. Consider a location-based application that provides querying facilities on geographical objects (e.g., airports, vehicles, and people) extracted from satellite images. Due to the errors incurred during satellite image transmission, the locations of the geographical objects can be imprecise. The data acquired from the Global Positioning System (GPS) and remote sensors can also be inaccurate and outdated, due to measurement error and network delay. During this paper, this paper tend to propose to live pattern frequentness supported the possible world linguistics. this paper tend to establish 2 unsure sequence information models abstracted from several real-life applications involving uncertain sequence information, and formulate the matter of mining probabilistically frequent serial patterns (or p-FSPs) from information that adapt to developed models. However the amount of attainable worlds is extraordinarily giant, that makes the mining prohibitively expensive. Impressed by the renowned PrefixSpan algorithmic program, this paper tends to develop 2 new algorithms conjointly referred to as UPrefixSpan.
\end{abstract}

\section{Keywords}

Frequent patterns, uncertain databases, approximate algorithm, GPS

\section{INTRODUCTION}

Data mining examinations the data gathered from diverse sources and gather valuable data from it. It discovers relationships or designs among handfuls of fields in substantia 1 social databases. It is exceptionally solid new innovation which causes organizations to center on most vital data from gathered data about their clients. It will be extremely effective innovation.

It will be exceptionally helpful to find any kind of data from pool of data. There are numerous sub range for scientists to work on it like bunching, order, continuous example mining, and so forth. Mining successive design will be most likely most vital idea of data mining. The example is called successive on the off chance that it happens numerous times in the exchange.

Analysts have given careful consideration to consecutive example mining on the grounds that it is utilized regularly. Consecutive design mining will be a subject of data mining concerned with discovering measurably pertinent examples between data samples where the values are conveyed in an arrangement. [4] Sequential design will be basically talked point in current time. Applications of consecutive design mining will be Medical medicines, common calamities, science \& designing methodologies, securities exchanges. For illustration, Customer shopping arrangements:

First purchase PC, then CD-ROM, and after that advanced cam, inside 3 months. Consecutive design mining calculations give this sort of valuable designs in exceptionally compelling way. So it is generally acknowledged truth be told application. In applications such as location-based services, natural habitat monitoring, web data integration, and biometric applications, the values of the underlying data are inherently noisy or imprecise. Consider a location-based application that provides querying facilities on geographical objects (e.g., airports, vehicles, and people) extracted from satellite images. Due to the errors incurred during satellite image transmission, the locations of the geographical objects can be imprecise. The data acquired from the Global Positioning System (GPS) and remote sensors can also be inaccurate and outdated, due to measurement error and network delay. As another example, consider a movie rating database integrated from WWW sources (e.g., IMDB movie database and user ratings obtained from the Netflix challenge). Due to the difference in the movie names specified in the sources (e.g., a user may type a wrong movie name), the integrated database may not be completely accurate. In biological applications, the extent of the area of a retina cell extracted from microscopy images can be inexact, due to limited image resolution and measurement accuracy.

In order to satisfy the increasing needs of the above applications, this paper envision that novel, correct, and scalable methods for managing uncertain data need to be developed.

To achieve this goal, this paper is leading for following steps:

Step 1: Develop a practical database system that incorporates uncertain data as a first-class citizen, in order to facilitate the development of the above applications; and

Step 2: Investigate the issues of data uncertainty in data mining, ambiguity removal, and data integration.

\section{Sequential Pattern Mining}

Sequential Pattern Mining is a new algorithm for finding all frequent sequences within a transactional database. The algorithm is especially efficient when the sequential patterns in the database are very long. A depth-first search strategy is used to generate candidate sequences, and various pruning mechanisms are implemented to reduce the search space.

The transactional data is stored using a vertical Column representation, which allows for efficient support counting as well as significant Column compression. A salient feature of our algorithm is that it incrementally outputs new frequent item-sets in an online fashion.

In a thorough experimental evaluation using standard benchmark data, this paper isolates the effects of the individual components of our algorithm. Our performance numbers show that our algorithm outperforms previous work by a factor of 3 to over an order of magnitude.

\section{PrefixSpan Algorithm}

Its major idea is that, instead of projecting sequence databases by considering all the possible occurrences of frequent 
subsequences, the projection is based only on frequent prefixes because any frequent subsequence can always be found by growing a frequent prefix.

\section{Apriori Algorithm}

Association rule generation is usually split up into two separate steps:

1. First, minimum support is applied to find all frequent itemsets in a database.

2. Second, these frequent itemsets and the minimum confidence constraint are used to form rules.

While the second step is straight forward, the first step needs more attention. Finding all frequent itemsets in a database is difficult since it involves searching all possible itemsets (item combinations). The set of possible item-sets is the power set over I and has size $2 n-1$ (excluding the empty set which is not a valid itemset). Although the size of the power set grows exponentially in the number of items $\mathrm{n}$ in I, efficient search is possible using the downward-closure property of support (also called anti-monotonicity) which guarantees that for a frequent itemset, all its subsets are also frequent and thus for an infrequent itemset, all its supersets must also be infrequent. Exploiting this property, efficient algorithms (e.g., Apriori and Eclat) can find all frequent item-sets.

Our contribution: this paper are using generalized sequential pattern mining algorithm. It is based on Apriori algorithm. The major strength of this algorithm is it generates candidates by Apriori pruning of database. GSP scans database multiple times. In the very first scanning all the items occurring in the database is counted and listed. From the sequence candidate 2- sequence is generated. [9][10] Now in next step support count of this candidate 2 -sequence is counted.

This candidate 2 -sequence will be the basis for next candidate 3 -sequence. This process is repeated until no more frequent sequence is found.

There mainly two major steps of the algorithm:

1. Candidate generation- which will generate the candidate sequence and perform join operation to perform next pass.

2. Support Counting. Normally, a hash tree-based search is employed for efficient support counting. Finally non-maximal frequent sequences are removed.

It uses the frequent items to iteratively project the sequence database in projected database while increasing subsequence's frequently. Each projection partitions the database and restricts further testing to smaller units. [11]

\section{LITERATURE SURVEY}

Information vulnerability is intrinsic in a few true applications like sensing component learning perception, RFID confinement and area based administrations, because of natural components, gadget restrictions, security issues, and so on. Thus, unsure information transforming has pulled in parts of consideration in late examination. The issue of mining Frequent Progressive Patterns (Fsps) from settled databases has pulled in loads of consideration in the examination group attributable to its wide range of world applications. as an illustration, in versatile pursue frameworks, Fsps is acclimated characterize then again group moving articles ; and in exploratory exploration, FSP mining helps find connections among grouping groupings. This paper tends to the matter of mining Fsps in the connection of unsure grouping information. [1]

The expanding occurrence of area procurement innovations such as GSM systems is prompt the gathering of huge spatialfleeting datasets and to the prospect of finding usable information about development conduct, which encourages fresh applications and administrations. This paper moves towards this bearing and create an expansion of the consecutive example mining standard that breaks down the trajectories of moving items. [2]

The huge greater part of the awhile ago created consecutive example mining techniques takes after the approach of Apriori which might significantly diminish the quantity of mixes to be analyzed. Be that as it may, Apriori still experiences issues when a progression database is vast and/or when repeated examples to be mined are various and/or long. Prexspan investigates prex-projection in successive example mining. Prexspan mines the complete set of examples however incredibly decreases the endeavors of hopeful subsequence era. Also, prex-projection munificently diminishes the extent of anticipated databases and prompts efficient handling. [3]

SPADE is another computation for fast discovery of Sequential Patterns. The existing answers for this issue make rehashed database outputs, and utilization complex hash structures which have poor territory. SPADE uses combinatorial properties to perish the first issue into littler sub issues that can be generously tackled in primary memory utilizing proficient grid look procedures, and utilizing basic join operations. All successions are established in just three database filters. Tests show that Spade out performs the best past calculation by a variable of two and by a request of greatness with some preprocessed information. [5]

\section{SYSTEM DESCRIPTION AND IMPLEMENTATION}

\subsection{Problem Definition}

Expected support as the measurement of pattern frequentness, which has inherent this weak-nesses with respect to the underlying probability model, and is therefore ineffective for mining high-quality sequential pat- terns from uncertain sequence databases.

\subsection{System Design}




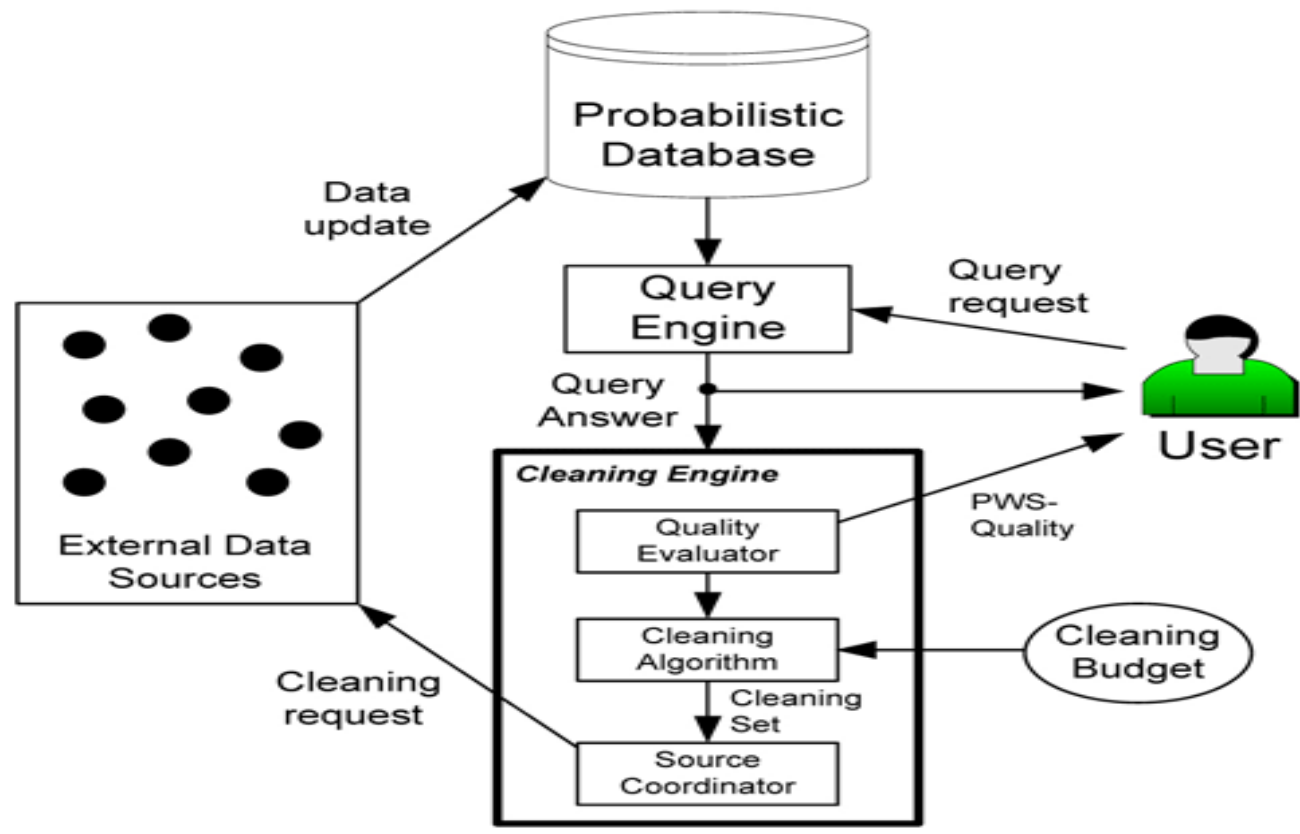

Figure 2.1: System Diagram

In uncertain sequence data mining, many real-world applications like sensor networks as well as customer purchase sequence. To mine frequent sequential patterns from uncertain data three different approaches of p-FSE are proposed in this paper they are:

1. The uncertain data is collected from external data sources.

2. An approximate approach which approximates the frequency of episode using probability models.

3. An optimized approach which efficiently prunes candidate episodes by estimating an upper bound of its frequentness probability using approximation techniques.

4. The cleaning engine is applying Prefixspan algorithm to mine the data item-sets.

FSE discovering is useful for mining frequent incident from sequential data. In frequent sequence pattern mining each element consist list of elements and each element consists of list of item symbolic, on the other hand frequent serial episode mines frequent subsequences from single long sequence of events. It consists of ordered list of uncertain events. To mine P-FSP over uncertain sequence, this paper has developed three mining algorithms. First approach discovers P-FSP by calculating accurate probabilities of episodes using dynamic programming. Secondly, approximate approach which approximates frequentness probabilities using probability models. Third, approximation approach which uses NP-Hard solution to give final result.

\subsection{Algorithm}

* Scan $S \mid \alpha$ once,

find the set of frequent items b such that:

- $\quad b$ can be assembled to the last element of $\alpha$ to form a sequential pattern; or

- $\quad<$ b $>$ can be appended to $\alpha$ to form a sequential pattern.

* For each frequent item b:

- $\quad$ append it to $\alpha$ to form a sequential pattern $\alpha^{\prime}$ and

output $\alpha^{\prime}$;

- $\quad$ output $\alpha^{\prime}$;

* For each $\alpha$ ':

- construct $\alpha^{\prime}$-projected database $\mathrm{S} \mid \alpha^{\prime}$ and

- call PrefixSpan $\left(\alpha^{\prime}, \mathrm{L}+1, \mathrm{~S} \mid \alpha^{\prime}\right)$.

\section{CONCLUSION}

In this paper, uncertainty will be exceptionally regular in all kind of databases. To address the issue of instability we have examined calculation related to it. We have centered on the calculation which mines consecutive design from unverifiable database.Past work utilizes help consider a premi se to take care of the issue. PrefixSpan is most broadly utilized calculation to tackle the issue. We have talked about various calculations like arrangement level U-PrefixSpan, p-FSE, Element level U- PrefixSpan and so forth all this calculation will be extremely helpful to mine consecutive example from questionable database. This paper looks forward to create calculations for incessant consecutive mining over questionable information.

\section{ACKNOWLEDGMENTS}

Our heartfelt thanks go to Dr. D. Y. Patil College of Engineering, Ambi for providing a strong platform to develop our skills and capabilities. This paper would like to thank to our guide \& respected teachers for their continuous support and incentive for us. Last but not least, this paper would like to thanks all those who directly or indirectly help us in presenting the paper.

\section{REFERENCES}

[1] M. Muzammal and R. Raman, "Mining sequential patterns from probabilistic databases", in Proc. 15th PAKDD, Shenzhen, China, 2011

[2] F. Giannotti, M. Nanni, F. Pinelli, and D. Pedreschi, "Trajectory pattern mining", in Proc. 13th ACM SIGKDD San Jose, CA, USA, 2007 
[3] D. Tanasa, J. A. Lpez, and B. Trousse, "Extracting sequential patterns for gene regulatory expressions proles", in Proc. KELSI, Milan, Italy, 2004.

[4] J. Pei et al., "PrexSpan: Mining sequential patterns efciently by prexprojected pattern growth", in Proc. $17^{\text {th }}$ ICDE, Berlin, Germany, 2001.

[5] R. Agrawal and R. Srikant, "Mining sequential patterns", in Proc. 11th ICDE, Taipei, Taiwan, 1995

[6] M.J.Zaki, "SPADE: An efficient algorithm for mining frequent sequences", Mach. Learn., vol. 42, no. 12, pp. $3160,2001$.

[7] J. Han, J. Pei, B. Mortazavi-Asl, Q. Chen, U. Dayal, and M. C. Hsu, "FreeSpan: Frequent pattern-projected sequential pattern mining", in Proc. 6th SIGKDD, New York, NY, USA, 2000.

[8] R. Srikant and R. Agrawal, "Mining sequential patterns: Generalizations and performance improvements", in Proc. $5^{\text {th }}$ Int. Conf. EDBT, Avignon, France, 1996

[9] Z. Zhao, D. Yan, and W. Ng, "Mining probabilistically frequent sequential patterns in uncertain databases", in
Proc 15th Int. Conf. EDBT, New York, NY, USA, 2012

[10] C. Gao and J. Wang, "Direct mining of discriminative patterns for classifying uncertain data", in Proc. 16th ACM SIGKDD, Washington, DC, USA, 2010.

[11] C. C. Aggarwal, Y. Li, J.Wang, and J.Wang, "Frequent pattern mining with uncertain data", in Proc. 15th ACM SIGKDD, Paris, France, 2009.

[12] Q. Zhang, F. Li, and K. Yi, "Finding frequent items in probabilistic data", in Proc. ACM SIGMOD, Vancouver, BC, Canada, 2008

[13] Nikos Pelekis, Ioannis Kopanakis, Evangelos E. Kotsifakos, Elias Frentzos "Clustering uncertain trajectories", 2010

[14] L. Sun, R. Cheng, D. W. Cheung, and J. Cheng, "Mining uncertain data with probabilistic guarantees," in Proc. 16th ACM SIGKDD, Washington, DC, USA, 2010.

[15] C. C. Aggarwal, Y. Li, J. Wang, and J. Wang, "Frequent pattern mining with uncertain data," in Proc. 15th ACM SIGKDD, Paris, France, 2009 